\title{
An Adaptive MAC Protocol for SDCS System Based on LoRa Technology
}

\author{
Tian Deng ${ }^{1, a}$, Jiang Zhu ${ }^{1, b}$, Zhiqiang Nie ${ }^{1, c}$ \\ ${ }^{1}$ Department of Electronic Science and Engineering, National University of Defense Technology, \\ Changsha, China
}

aemail: dengtian_nudt@163.com, bemail: jiangzhu@nudt.edu.cn, 'email: niezq_nudt@163.com

Keywords: LoRa; SA-LoRaWAN; SDCS; Energy-efficient

\begin{abstract}
The sensor data collection satellite (SDCS) system, as a part of the Internet of Things (IoT), enables data transmission in areas without Internet and mobile communication coverage. A novel technology named LoRa for Low power wide area network (LPWAN) enables energy-efficient communication over long distances, which can meet the requirement of energy-constraint in SDCS system. However, the LoRaWAN as the MAC protocol of LoRa has the problem of initial channel allocation and power selection if directly used in SDCS system. In this paper, we propose a SA-LoRaWAN protocol for power adaptation based on position information of sensors and satellite, and sensors can dynamically access channel according to the packet buffer size. We use OPNET to model SDCS system based on SA-LoRaWAN protocol, and the simulation results demonstrate that the proposed protocol can reduce the energy consumption and improve the packet delivery rate.
\end{abstract}

\section{Introduction}

Nowadays, the demand for collecting data by satellite is more and more significant in the era of large data. The SDCS system, which combines the LEO satellite and the IoT terminals with sensing and information gathering function, can be used to build a global coverage information acquisition and transmission system. The system will meet the communication needs of dispersed terminals in harsh geographical environment, provide users with long distance communication services, realize the real-time return of global situational awareness information, and solve the problems of low efficiency of various kinds of perceptual information feedback.

LoRa (Long Range) [1] is a linear spread spectrum technology proposed for LPWAN with the ultra-long transmission distance [2]. Experiments show transmission ranges of up to $1.3 \mathrm{~km}$ in an urban environment with a frame loss of $10 \%$ [3], and of up to $30 \mathrm{~km}$ in sea area with loss ratio $32 \%$ [4]. LoRaWAN is a LPWAN standard based on the open source MAC layer protocol introduced by the LoRa Alliance. [5] [6] show the performance and throughput of LoRaWAN network. It is expected that the application of the LoRaWAN to the SDCS system will increase the lifetime of the terminal and improve the system capacity and quality of service (QoS) under the energy limited.

The remainder of this paper is organized as follows. First we take a brief look on the physical layer and MAC layer of LoRa technology. Then we design SA-LoRaWAN protocol for SDCS system. And we use OPNET to establish a model including satellite orbit, wireless pipeline and node model. Finally, we simulate and discuss the performance of SA-LoRaWAN.

\section{The LoRa Technology}

The $L o R a^{T M}$ as the physical layer technology of LoRa uses a chirp spread spectrum (CSS) modulation, which realizes the spread spectrum communication by matching filtering and pulse compression characteristic of chirp signal, instead of using a pseudo-random sequence. The instantaneous frequency of the chirp signal is linear time-varying, and the phase between different chirp symbols is continuous. This enables receiver to achieve time and frequency synchronization simply.

CSS modulation not only has the advantage of traditional spread spectrum technology, but also 
has high resistance of interference, Doppler shift and multi-path fading with low transmit power in mobile communications. It combines low complexity and low power consumption characteristics, and the signal can be demodulated below noise floor, which improves the sensitivity of LoRa receiver and increases the link budget.

The sensor nodes can choose the spread spectrum modulation bandwidth (BW), spreading factor (SF) and error correction rate (CR) automatically. LoRa ${ }^{T M}$ supports three kinds of bandwidth, which is $B W \in\{125,250,500\} \mathrm{KHz}$. Increasing the signal bandwidth can effectively improve the data rate, but lead to lower sensitivity. Chip rate is $R_{\mathrm{c}}=B W$, the SF is the ratio between the chip rate and the symbol rate, indicating the number of symbols transmitted per information bit, as $S F=\log _{2}\left(\mathrm{R}_{c} / \mathrm{R}_{s}\right)$. It defines 6 spreading factors $S F \in\{7, \ldots, 12\}$, different SFs are orthogonal distribution, so multiple transmission signals can occupy on a single channel. In order to further improve the robustness of the link, the $L o R a^{T M}$ modem employs cyclic error coding as RateCode $=\frac{4}{4+R}$ to perform forward error detection and correction, where $R \in\{0,1,2,3,4\}$. Therefore, the effective data rate is given by

$$
R_{b}=S F \frac{\text { RateCode }}{2^{S F} / B W}[\mathrm{bit} / \mathrm{s}]
$$

According to [7], when $B W=125 \mathrm{KHz}$, RateCode $=4 / 5$, the parameters of different SFs are shown in Table 1.

Table 1 The parameters corresponding to different spreading factors

\begin{tabular}{|l|l|l|l|l|}
\hline $\begin{array}{l}\text { Configuratio } \\
\mathrm{n}\end{array}$ & Chips/Symbol & Bit Rate(bit/s $)$ & Demodulator SNR & Receiver Sensitivity \\
\hline LoRa:SF12 & 4096 & 293 & $-20 \mathrm{~dB}$ & $-137.5 \mathrm{dBm}$ \\
\hline LoRa:SF11 & 2048 & 537 & $-17.5 \mathrm{~dB}$ & $-135 \mathrm{dBm}$ \\
\hline LoRa:SF10 & 1024 & 977 & $-15 \mathrm{~dB}$ & $-132.5 \mathrm{dBm}$ \\
\hline LoRa:SF9 & 512 & 1758 & $-12.5 \mathrm{~dB}$ & $-130 \mathrm{dBm}$ \\
\hline LoRa:SF8 & 256 & 3125 & $-10 \mathrm{~dB}$ & $-127.5 \mathrm{dBm}$ \\
\hline LoRa:SF7 & 128 & 5469 & $-7.5 \mathrm{~dB}$ & $-125 \mathrm{dBm}$ \\
\hline
\end{tabular}

LoRaWAN defined by LoRa alliance is a protocol based on LoRa technology, which mainly includes three parts: LoRa terminal, LoRa gateway and LoRa server. The terminal and the gateway connect with the star topology, and the gateway and the server are connected by the standard IP. All devices support two-way communication, but mainly the uplink.

LoRaWAN specification 1.0.2 [8] divided the terminal equipment into A / B / C types according to the actual application. Class- $\mathrm{A}$ is the lowest power consumption terminal. The terminal sends packets in ALOHA access mode based on its own requirements. Each terminal opens two short downlink receive windows after uplink transmission. The rest of the time is in a sleep state. A periodic beacon sending by the gateway is used to synchronize the Class-B devices to allow scheduling of additional downlink transmissions. This mode need to monitor the information, so power consumption is greater than Class-A. Finally, Class-C end-devices with maximal receive slot are always listening to the channel except when they transmit.

LoRaWAN supports six data frame types and 18 MAC commands for server-to-terminal MAC layer interaction. LoRa network allows the terminal adaptive data rate (ADR) individually. Formula (1) shows the transmission rate, which is determined by BW, SF and CR. When BW and CR is fixed, small SF represents high data rate and short transmission time, but the sensitivity will be reduced accordingly. When the terminal is far away from the gateway, the high SF can improve the robustness and the transmission distance, but a low rate. The key factors that affect system capacity are the number of concurrent channels, the data rate, the length of the load, and how the node sends data. 


\section{SA-LoRaWAN Protocol for SDCS System}

As is well know, the one of the key factors for wireless sensor network (WSN) performance is low power consumption, so it is necessary to design a MAC protocol to save the node energy. In this section, we propose a MAC protocol for SDCS system, called SA-LoRaWAN, which is designed to guarantee energy-efficient, timely data delivery and reliable LEO satellite communication. Suppose system parameters of the LEO satellite are as follows: the orbit height is $480 \mathrm{~km}$, the flight period is about 1.5 hours, and the cross-time is about 8 minutes. The satellite orbital position can be calculated by the six parameters of major semi-axis $a$, eccentricity $e$, mean anomaly $M_{0}$, argument of perigee $\omega$, orbit inclination $i$, right ascension of the ascending node $\Omega$. In the SDCS system, the distance between satellite and ground sensor is range from 480 to $1600 \mathrm{~km}$ which may lead to unnecessary waste if a constant power transmission scheme were used. Simultaneously, satellite over the top will trigger a vast number of sensors, so the collision is serious in the initial channel assignment, thereby causing the "avalanche" problem. It may cause failure of activation of most sensors, and meanwhile the increase of system latency. To solve these problems, we propose a SA-LoRaWAN protocol to improve the system performance.

To improve the energy efficient, we propose a power and data rate adaptation mechanism. First, the sensor in SDCS system can calculate the satellite cross-time and the transmission distance based on position information, and the current minimum transmission power can be calculated according to the free space propagation loss formula $L_{s}=92.44+20 \log d(\mathrm{~km})+20 \log f(\mathrm{GHz})$, where $d$ represent transmission distance, when the performance of uplink transmission reach the link margin. Second, the SDCS system allows the sensors to individually use any of possible data rates, and each sensor will be optimized to use the fastest data rate possibly according to the network Quality of Service (QoS).

To resolve the collection problem during the initial channel allocation period of LoRaWAN when a large number of nodes activate at same time, the SA-LoRaWAN protocol also adds the function of activation percentage control mechanism. We suppose there are $N_{i}$ packet buffers in the node, where $i=(0, \ldots, 10)$ subjects to uniform distribution and $N_{\max }=10$. In order to ensure that node buffering more packets with higher priority, each node generates a random number $y=\operatorname{rand}\left(N_{i}-1, N_{i}\right)$ when it triggers. During the $t_{j}(j=(1, \ldots, 10))$ minute, the join request will be sent when the condition $y \geq\left(N_{\max }-t_{j}\right)+0.5$ is satisfied. Otherwise, the sensor sends the join request after random delay during the $t_{j}$ minute when $\left(N_{\max }-t_{j}\right)+0.5>y>\left(N_{\max }-t_{j}\right)$, and the sensor do not operate during the $t_{j}$ minute and waits for the next minute when $y \leq\left(N_{\max }-t_{j}\right)$. During the last minute of the satellite cross-time, all nodes can send packets regardless of whether the generated random number satisfies the launch condition. Through this distribution criterion, we disperse the sensor's network activation during the communication time to avoid overcrowding of the channel.

Thus, the SA-LoRaWAN follows the basic process of LoRaWAN, and can be summarized as shown in Figure 1. For each upstream transmission, the sensor selects a channel and SF at random. And the downlink frequency is the same as the uplink frequency. The satellite calculates the remaining time after receiving the packet, and then transmits when the receive window is opening. If the time of window 1 has elapsed, it can choice window 2 to transmit the packet. Retransmission can occur only after timeout of ACK timer. The retransmission packet will select another channel and a large SF to increase the robustness. After reach the maximum number of retransmissions, the sensor can decide whether to retransmit or discard the packet. Downstream data usually send through ACK bearer. When there are consecutive downlink packets need to deliver, it only can through the frame-pending mechanism shown in Figure 2 to reduce the downlink transmission delay. The "FPending" bit can only be set on a downlink frame which informs the sensor that the satellite has several pending frames. The node receives the packet will send an uplink packet as soon as possible, and it is best to send an empty message to open the next receive window. 


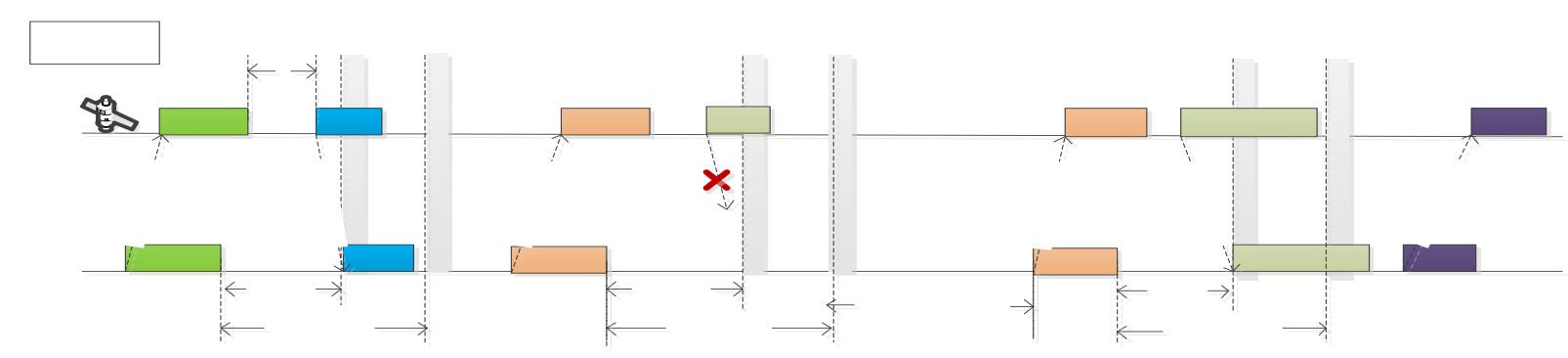

Fig.1. Class-A protocol

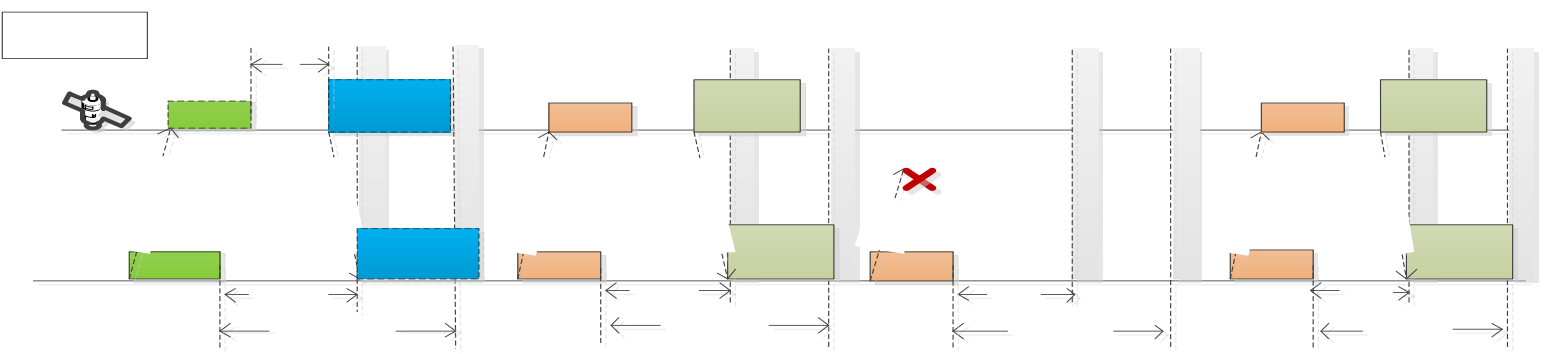

Fig.2. Fpending mechanism

\section{System Modeling and Simulation Verification}

\section{SDCS System Modeling.}

In this section, we model the SDCS system based on OPNET. The trajectory of the satellite is imported from the STK track file, and OPNET through 14 pipeline stages to simulate packet transmission in the wireless environment. In order to adapt to the SDCS system, we change the default pipeline appropriately. Due to the high-speed movement of the satellite, the network has a dynamic topology. On Stage0 (receiver group), we dynamic binding transmitter-receiver to update the receiver group periodically. Stage7 (received power) has a global Boolean variable named "singal_lock" that prevents packets from being received at the same time, so we remove it to allow packets of different SFs can be simultaneously demodulated on the same channel. During Stage11, OPNET calls parameters of modulation curve, the signal noise ratio (SNR) and processor gain are computed in the Stage 10 to obtain the effective SNR. OPNET invokes only one modulation curve a time as default. However, different SF corresponds to different receiver sensitivities. We refer to Table 1 parameters to change the pipeline so that different SF calls different modulation patterns.

Each sensor uses Bei-Dou satellite positioning system and has a built-in satellite ephemeris data. That is, the position of satellite is known to sensors. Sensors and satellite organize in a star-of-stars topology, and work in half-duplex mode by using LoRa technology to reduce energy consumption and hops. We also assume that the satellite combines function of LoRa gateway with LoRa server. The sensor and satellite model has shown in Figure 3 (a) and (b), respectively. Because we focus on the study of access protocols, we simplify the MAC layer above as a signal source and a signal collection module. Both of sensors and the satellite have an uplink and a downlink. Compared to satellite, the sensor adds access control module for network activation and transmission power control, data rate adaptation and so on. 


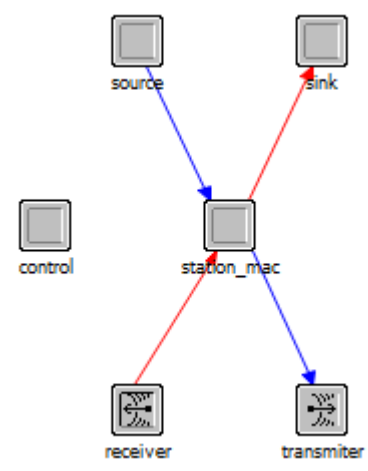

(a) Sensor node module

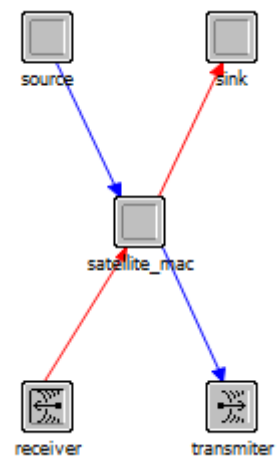

(b) Satellite node module

Fig.3. SDCS node module

\section{Simulation Verification.}

The system parameters are shown in Table 2, and we assume that the packet size and the number of packet buffers are uniformly distributed. In simulation, we tests the energy consumption by using different SFs, under conditions that whether to use the power adaptive mechanism. And we also compare the packet delivery ratio on different scenarios.

Table 2 Main simulation parameters

\begin{tabular}{cc}
\hline Simulation parameters & Parameter value \\
\hline Satellite height & $480 \mathrm{~km}$ \\
Number of sensors & $100 \sim 800$ \\
Satellite coverage diameter & $3000 \mathrm{~km}$ \\
Simulation time & $480 \mathrm{~s}$ \\
Data rates & $293 \mathrm{bit} / \mathrm{s} \sim 5469 \mathrm{bit} / \mathrm{s}$ \\
Transmission power & $1 \mathrm{~W} \sim 2 \mathrm{~W}$ \\
Packet size & $100 \sim 500 \mathrm{bits}$ \\
Packet buffers & $0 \sim 10$
\end{tabular}

Figure 4 shows the distribution of different SFs when sensors use ADR mechanism to communicate with satellite. We can see that the SF7 accounts for 55\%, while the SF11 only takes $4 \%$. It indicates that the sensor tends to select a small SF to save the transmission time, thereby reducing energy consumption.

Figure 5 represents the total energy consumption for SDCS system by using constant power and location-based power control. It can be seen from the figure that the power consumption based on position information during satellite cross-time is significantly lower than that using constant power. For location-based power control, the energy consumption of fixed spreading factors of SF7 and SF10 are compared to ADR mechanism, the results show that the energy consumption of ADR is close to the performance of SF7 and much better than the performance of SF10, due to the probability of packet collision is increased when SF10 is selected. As the number of nodes increases, the rate of energy consumption drastically increases.

A comparison of the delivery ratio with or without activation rate control is shown in Figure 6 . When the number of nodes is few, the delivery rate of two protocols is similar and reaches the peak value above 0.9. As the number of nodes increases, the delivery ratio decrease quickly in LoRaWAN due to a large number of nodes trigger at the same time, but the proposed protocol that controls sensor nodes to scatter communication during satellite cross-time can reduce the flow pressure. It can be seen when the number of nodes reaches 800 , the SA-LoRaWAN achieves 70\% packet delivery ratio, while LoRaWAN only about $20 \%$. We can see that the mechanism with ADR and power control balances the performance of energy consumption and the packet delivery ratio, 
and obtain the best comprehensive performance.

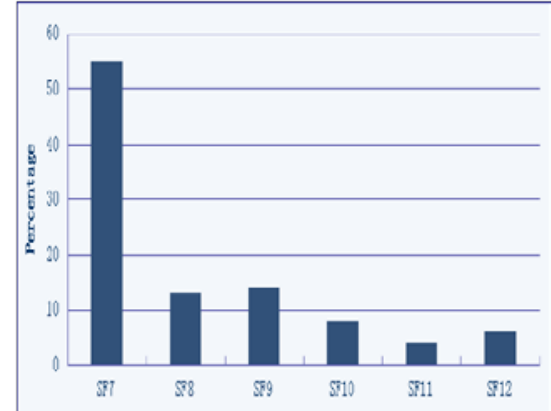

Fig.4. SF Distribution Table

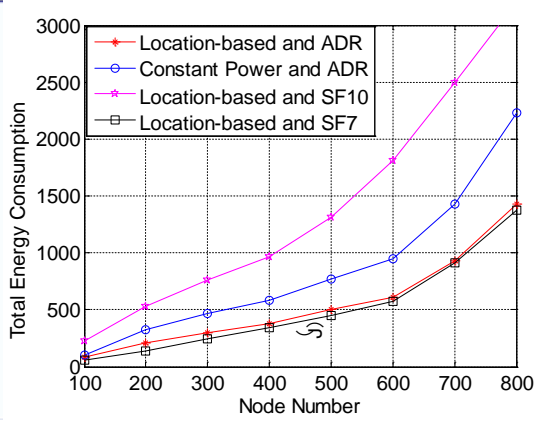

Fig.5. Energy Consumption

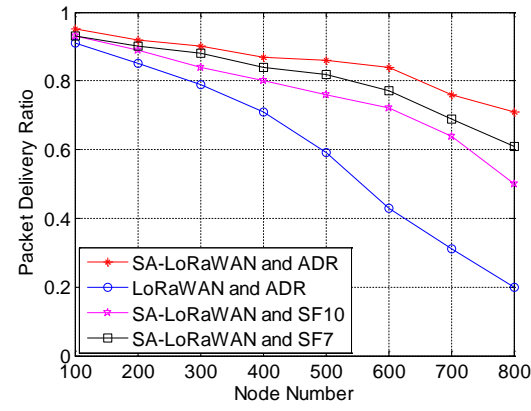

Fig.6. Delivery Ratio

\section{Conclusion}

In this paper, we proposed SA-LoRaWAN as an energy-efficient adaptive access MAC protocol, for SDCS system. We have implemented SA-LoRaWAN in SDCS system by using OPNET and evaluated its performance. Simulation results demonstrate that SA-LoRaWAN can effectively reduce the sensor energy consumption, and improves the success rate of packet delivery by reducing the congestion degree of the channel. Thus, It is expected that the SDCS system based on SA-LoRaWAN will increase the lifetime of the terminal and improve the system capacity and quality of service (QoS) under the energy limited.

\section{References}

[1] Semtech. $L o R a^{T M}$ modulation basics, Semtech Corporation, application note 1200.22, May 2015, revision 2.

[2] Link Labs. A Comprehensive Look At Low Power, Wide Area Networks For Internet of Things Engineers and Decision Makers, White Paper, 2016.

[3] Centenaro M, Vangelista L, Zanella A, et al. Long-range communications in unlicensed bands: the rising stars in the IoT and smart city scenarios [J]. Computer Science, 2015, 23.

[4] Petajajarvi J, Mikhaylov K, Roivainen A, et al. On the coverage of LPWANs: range evaluation and channel attenuation model for LoRa technology [C]. International Conference on ITS Telecommunications. IEEE, 2015:55-59.

[5] Adelantado F, Vilajosana X, Tusetpeiro P, et al. Understanding the limits of LoRaWAN [J]. 2016.

[6] Mikhaylov K, Juha Petaejaejaervi, Haenninen T. Analysis of Capacity and Scalability of the LoRa Low Power Wide Area Network Technology [C], European Wireless 2016, European Wireless Conference. VDE, 2016.

[7] LoRa Semtech. “SX1276/77/78 Data sheet,” Wireless, Sensing and Timing.

[8] Sornin N, Luis M, Eirich T, Kramp T, and Hersent O. LoRaWAN Specification, LoRa Alliance, Standard V1.0.2, 2016. 